\title{
Role of upper gastrointestinal investigations in a screening study for colorectal neoplasia
}

\author{
W M Thomas, J D Hardcastle
}

\begin{abstract}
Should patients with positive faecal occult blood screening tests who are free of colorectal neoplasia undergo upper gastrointestinal investigation? Altogether 16985 faecal occult blood tests were completed in a group of 18818 asymptomatic patients (45-75 years) offered screening at two yearly intervals. A total of 447 $(2 \cdot 6 \%)$ were positive and underwent large bowel investigations. No neoplastic disease was identified in $283(63 \%)$ of them. Fourteen $\mathbf{5 \%}$ ) also underwent gastroscopy for upper gastrointestinal symptoms, benign conditions were identified in five and a gastric carcinoma in one. No further investigations were instituted in the remaining 269 subjects who have now been followed up for a median period of 5 years (2-8 years). Five have been referred for benign upper gastrointestinal conditions, but none for upper gastrointestinal malignancy. Thirty one subjects have died - one from gastric cancer (a patient who had undergone a previous partial gastrectomy for a duodenal ulcer and who had persistent upper gastrointestinal symptoms). The remaining deaths were unrelated to the upper gastrointestinal tract. Nineteen people who have left the trial area have been monitored for the development of malignant disease; none have presented with upper gastrointestinal malignancy. These data support the view that upper gastrointestinal investigations need not be performed routinely in this group of subjects, but may be reserved for those with relevant symptoms.
\end{abstract}

Most patients with colorectal carcinoma present at a stage when the disease has spread to regional lymph nodes or has metastasised to distant organs. ${ }^{1}$ This is considered to be a major factor in the overall poor prognosis after treatment. ${ }^{23}$ The aim of screening for large bowel cancer is to detect the disease in its presymptomatic phase, when it is likely to be less advanced and curative surgical resection is possible.

Early results from the randomised trials of faecal occult blood screening are encouraging. It has been established that asymptomatic colorectal cancer can be detected by simple tests for faecal occult blood; furthermore these cancers are generally at a less advanced pathological stage than those occurring in the control populations. ${ }^{46}$

The test which has been most extensively studied, Haemoccult, has been shown to have a sensitivity for carcinoma of $45-74 \%^{46}$ and, considering a diagnosis of carcinoma or adenoma to be a true positive, a specificity of $98-99 \%$ in asymptomatic subjects. Feca EIA, a test which combines a chemical test of increased sensitivity for blood with a human-specific immunological test, has also been used in a cohort of patients in the Nottingham study. It has been shown to have a higher sensitivity for neoplasia but a correspondingly lower specificity, resulting in a higher rate of false positive reactions. ${ }^{78}$ While accepting that the specificity for neoplasia of both tests is high, when applied to the large numbers involved in mass population screening an appreciable number of people will be shown to be free of colorectal neoplasia after investigation of the large bowel. In most of these no satisfactory cause for the positive test will be apparent.

Healthy people may lose gastrointestinal blood in sufficient amounts to result in a positive faecal occult blood test ${ }^{9}$; furthermore dietary factors may be responsible for false positive results. ${ }^{10}$ Nevertheless, failure to identify a colonic source of bleeding in subjects with positive faecal occult blood tests poses a question of great practical and ethical importance - should they undergo investigation of the remainder of the gastrointestinal tract to exclude pathology at sites other than the colorectum?

We have aimed to answer this question by reviewing the subjects in whom no colonic neoplasia was identified after investigation of positive faecal occult blood tests in a large randomised study of screening for colorectal neoplasia.

Given the high prevalence of diverticular disease " and benign perianal conditions (in particular haemorrhoids) ${ }^{12}$ in this age group, we have not considered either of these diagnoses as a satisfactory explanation of a positive faecal occult blood test.

\section{Method}

Asymptomatic subjects between 50 and 74 years of age were identified from general practitioner lists. After stratification by age and sex they were randomly allocated to a test group who received faecal occult blood tests or to a control group who were not approached.

After completion, the tests were interpreted by experienced staff and those with positive tests were seen in a designated early diagnosis clinic. A full history, including upper gastrointestinal symptoms, was taken. Subjects subsequently underwent either colonoscopy or flexible sigmoidoscopy and double contrast barium enema.

Upper gastrointestinal investigations were not performed routinely, but subjects with relevant symptoms - for example, dyspepsia or dysphagia - were referred for gastroscopy.

Subjects with negative tests, or in whom colonic investigations were normal, were 


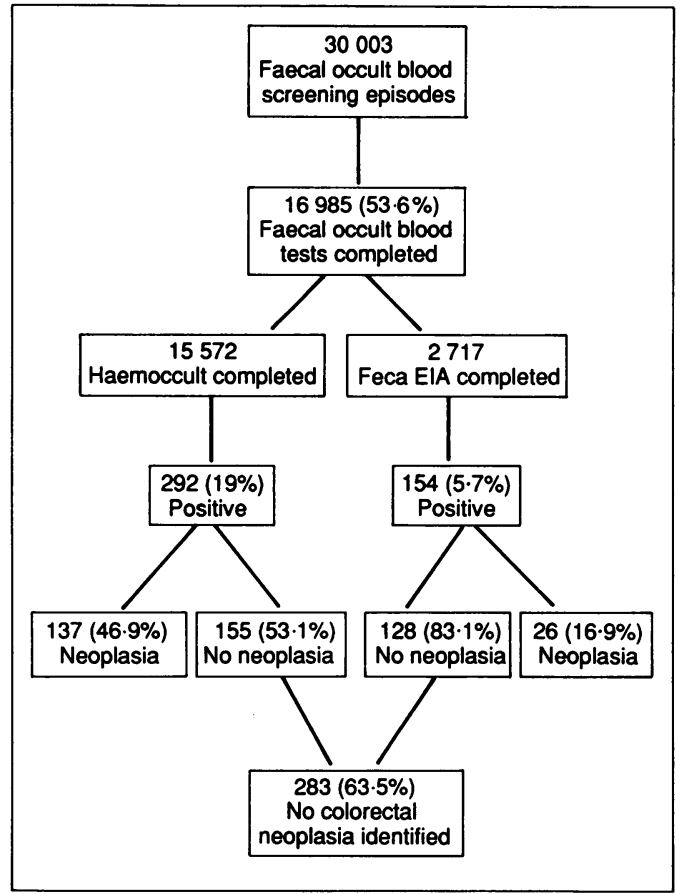

Figure 1: Identification of neoplasia in subjects undergoing large bowel investigation following positive faecal occult blood tests.

rescreened at intervals of two years; they were also monitored for the development of colorectal neoplasia by scrutiny of the computerised pathology records of the Nottingham hospitals and by information supplied by the Trent Cancer Registry and the National Health Service central registry at Southport.

Furthermore, the case notes or microfilm records of subjects who had been referred to the Nottingham hospitals were reviewed. A total of 257 subjects had hospital records; in 36 of these the hospital episode was related solely to the screening study. A further 221 patients also had records relating to other outpatient referrals or hospital admissions and of these the hospital records of 211 were available for review.

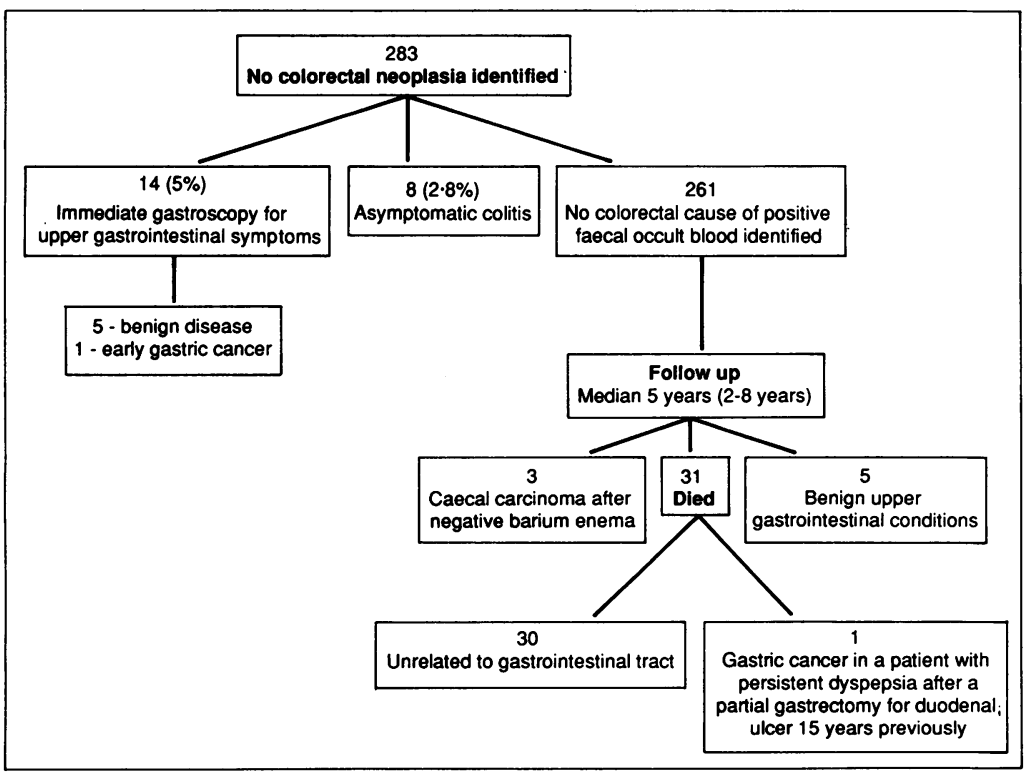

Figure 2: Outcome in 283 people with positive faecal occult blood tests in whom no colonic neoplasia was identified.
We have considered the diagnosis of colorectal carcinoma or histologically proved adenoma to be a true positive faecal occult blood test. In addition, conditions known to produce colonic bleeding - for example, ulcerative colitis - have been recorded.

In general, Haemoccult was the screening test used, but some subjects were screened with Feca EIA.

\section{Results}

A total of 18818 subjects were offered faecal occult blood tests and those who complied were offered future tests at two yearly intervals. Altogether 7644 have been rescreened once and 3541 rescreened twice, a total of 30003 screening episodes (Fig 1). Overall $16985(56 \cdot 6 \%)$ of tests were completed $(8123(43 \cdot 2 \%)$ at the initial screen, $5976(78 \cdot 2 \%)$ at the first rescreen, and $2886(81 \cdot 5 \%)$ at the second rescreen.

Some 15572 subjects completed Haemoccult and 2717 completed Feca EIA tests (1304 completed both tests). A total of $292(1.9 \%)$ of the Haemoccult tests were positive for faecal occult blood. Further investigation showed neoplastic disease in $137(46.9 \%)$ subjects and no neoplastic disease in $155(53 \cdot 1 \%)$. The rate of positive reactions and yield of neoplasia of Feca EIA in comparison with Haemoccult has been reported previously..$^{78}$ Of the 2717 participants who completed Feca EIA tests, $154(5 \cdot 7 \%)$ were positive. Investigation of these showed neoplastic disease in $26(16.9 \%)$ and no neoplastic disease in 128 $(83 \cdot 1 \%)$.

Overall, of the 446 subjects with positive tests, colorectal neoplasia was not identified in 283 $(63 \cdot 5 \%)$ (Fig 2). Fourteen (5\%) of these also underwent gastroscopy for upper gastrointestinal symptoms, benign gastroduodenal disease being identified in five and an early gastric cancer in one. Four patients with chronic, intermittent dyspepsia were not investigated.

No further investigations were instituted in the remaining 269 subjects.

In seven of these patients there was endoscopic and histological evidence of ulcerative colitis or colonic Crohn's disease, and of a radiation colitis (secondary to treatment of a carcinoma of the uterine cervix) in one. These were considered to be the cause of the positive faecal occult blood tests, leaving 261 subjects in whom no satisfactory cause for blood loss was identified. These have now been followed up for a median period of 5 (range 2-8) years.

During this time a diagnosis of benign upper gastrointestinal disease has been made in five subjects after the investigation of symptoms that have developed since the patients were screened.

Three patients who had negative double contrast barium enema examinations subsequently presented with large bowel carcinoma (affecting the caecum in all cases) in the interval between the examinations and a rescreen at two years; these are presumed to have been missed by barium enema examination.

A total of 31 subjects have died - one of gastric carcinoma (a patient who had undergone a previous partial gastrectomy for duodenal ulcer). The remaining deaths were unrelated to the 
upper gastrointestinal tract, the principal causes of death being secondary to cardiovascular disease (11 patients) or respiratory disorders (seven patients).

Nineteen patients who have left the trial area have been monitored for the development of malignant disease, but none have presented with gastrointestinal malignancy.

\section{Discussion}

Total visualisation of the colorectal mucosa by radiological or endoscopic means is mandatory in screened subjects with positive faecal occult blood tests.

In the Nottingham study colonoscopy is currently the investigation of choice as it allows endoscopic resection of polypoidal lesions at the time of the examination. Double contrast barium enema examination is used to supplement colonoscopy in those in whom the caecum is not reached, and as a first line investigation in patients with severe respiratory disease.

In the early part of the study (on which much of the present data are based) double contrast barium enema combined with flexible sigmoidoscopy were used as the initial investigation and 26 carcinomas were thus detected. It seems probable that a further three carcinomas were not identified, however, resulting in a sensitivity of $89 \cdot 7 \%$ for large bowel malignancy.

The prevalence of asymptomatic inflammatory bowel disease has been previously reported. ${ }^{13}$ Given the importance of surveillance of colitis this must be viewed as a positive aspect of the screening programme. In each of the eight subjects mentioned, there was macroscopic evidence of inflamed mucosa and it is therefore reasonable to assume that this was the cause of the positive faecal occult blood tests.

The extent to which the remainder of the gastrointestinal tract should be investigated in the remaining subjects in whom no satisfactory colorectal cause for bleeding has been found is controversial. In practical terms this would involve routine upper gastrointestinal endoscopy, possibly followed by a small bowel barium enema. Even then rare causes of blood loss, such as haemobilia, would be missed.

It may be argued that having identified an asymptomatic subject with a positive faecal occult blood test, one has an obligation to investigate fully the gastrointestinal tract if the colorectum is normal. However, Haemoccult and the more recently developed immunological tests have been shown to be relatively poor indicators of upper gastrointestinal abnormalities. ${ }^{14}$ is This is probably a reflection of the degradation of blood that occurs during colonic transit. We have shown previously that Haemoccult is significantly less sensitive for caecal cancers than for cancers of the sigmoid or descending colons. ${ }^{16}$ If this is a reflection of gastrointestinal degradation, one would expect an even greater effect on blood from gastric or duodenal lesions.

An alternative strategy is to reserve upper gastrointestinal endoscopy for those patients with relevant symptoms. Whereas early colorectal neoplasia is generally an asympto- matic condition, there is evidence that gastric carcinoma produces dyspeptic symptoms in a significant proportion of subjects. ${ }^{17}$ In a programme for the early detection of gastric cancer, a yield of $3 \cdot 4 \%$ for oesophageal and gastric cancer was obtained in subjects over the age of 40 years presenting to their general practitioners with dyspeptic symptoms. ${ }^{18}$ It is evident that this group of individuals should undergo investigation. Of the 14 subjects in the present study who underwent upper gastrointestinal endoscopy, five $(35 \cdot 7 \%)$ were shown to have benign gastroduodenal disease and one to have an early gastric carcinoma. It is therefore mandatory that upper gastrointestinal symptoms should be sought in Haemoccult positive subjects in whom large bowel investigations are normal.

Of the four patients with chronic dyspepsia, one who was not investigated was diagnosed as having a gastric cancer some 24 months after a negative large bowel examination. In retrospect, this symptomatic patient should have undergone gastroscopy after large bowel investigation and this subject emphasises the importance of investigating those with upper gastrointestinal symptoms.

The remaining patients, who did not report upper gastrointestinal symptoms, have now been followed up for a median period of five years. The only gastrointestinal conditions which have been identified to date have been minor degrees of gastritis or oesophagitis in five patients, and it seems unlikely that there was an important gastrointestinal cause of blood loss at the time of the positive faecal occult blood test. In particular, we are reassured that no subjects in this group have presented with upper gastrointestinal malignancy and conclude that upper gastrointestinal investigations need not be performed routinely in screened subjects who have positive tests, but should be reserved for those with relevant symptoms.

1 Stower MH, Hardcastle JD. The results of 1115 patients with colorectal cancer treated over an 8 year period in a single hospital. Eur f Surg Oncol 1985; 11: 119-23.

2 Miller AB. Trends in cancer mortality and epidemiology. Cancer $1983 ; 51: 2413-8$.

3 Slaney G. Results of treatment of carcinoma of colon and rectum. Mod Trends Surg 1971; 3: 69-89.

4 Kewenter J, Bjork S, Haglind E, Smith L, Svanvik J, Ahren C. Screening and rescreening for colorectal cancer. A C. Screening and rescreening for colorectal cancer. A controlled trial of faecal occult
subjects. Cancer 1988; 62: 645-51.

5 Kronberg $\mathrm{O}$, Fenger $\mathrm{C}$, Sondergaard $\mathrm{O}$, et al. Initial mass screening for colorectal cancer with faecal occult blood test. Scand $\mathcal{F}$ Gastroenterol 1987; 22: 677-86.

6 Hardcastle JD, Thomas WM, Chamberlain J, et al. Randomised, controlled trial of faecal occult blood screening for colorectal cancer: results for first 107349 subjects. Lancet 1989 ; i: $1160-4$.

7 Pye G, Ballantyne KC, Armitage NC, Hardcastle JD Comparison of three day haemoccult, six day haemoccul and Fecatwin/Feca ElA Tests for the detection of faecal occult blood in screening for colorectal cancer [Abstract] Gut 1986; 27: A1242.

8 Armitage NC, Hardcastle JD, Amar SS, Balfour TW, Haynes J, James PD. A comparison of an immunological faecal J, James PD. A comparison of an immunological faecal occult blood test Fecatwin sensitive/Feca ElA with haemoccult in population scre

9 Stroehlein JR, Fairbanks VF, McGill DB, et al. Haemoccul Stroehlein JR, Fairbanks VF, McGill DB, et al. Haemoccult Am f Dig Dis 1976; 21: 841-4

10 Bassett ML, Goulston KJ. False positive and negative haemoccult reaction on a normal diet and effect of die restriction. Aust NZ J Med 1980; 10: 1-4.

11 Painter NS, Burkitt DP. Diverticular disease of the colon, 20th century problem. Clin Gastroenterol 1975; 4: 3-22.

12 Haas PA, Haas GP, Schmaltz S, Fox TA. The prevalence of hemorrhoids. Dis Colon Rectum 1983; 26: 435-9.

13 Mayberry JF, Ballantyne $\mathrm{KC}$, Hardcastle $\mathrm{JD}$, et at. 
Epidemiological study of asymptomatic inflammatory bowel disease: the identification of cases during a screening programme for colorectal cancer. Gut 1989; 30: 481-3.

14 Ahlquist DA, McGill DB, Schwartz S, Taylor WF. Faecal blood levels in health and disease. A study using HemoQuant. $N$ Engl F Med 1985; 312: 1422-8.

15 McDonald CA, Walls RS, Burford Y Goulston KJ, Yuen AC. Immunochemical detection of fecal occult blood. Aust $N Z \mathcal{Y}$
Med 1984; 14: 105-10.

16 Thomas WM, Pye G, Hardcastle JD. Site-dependent sensitivity of Haemoccult screening: implications for test design. Br F Surg 1989; 176: 1346.

17 Oleagoitia JM, Echevarria A, Santidrian JI, et al. Early gastric cancer. Br F Surg 1986; 73: 804-6.

18 Allum WH, Hallissey MT, Dorrell A, et al. Programme for early detection of gastric cancer. $\mathrm{Br}$ Med $\mathcal{F}$ 1986; 293 : 541 . $\stackrel{\rho}{\rightleftharpoons}$

$\vec{\Rightarrow}$ 